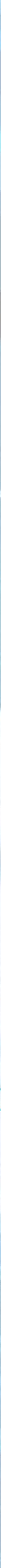



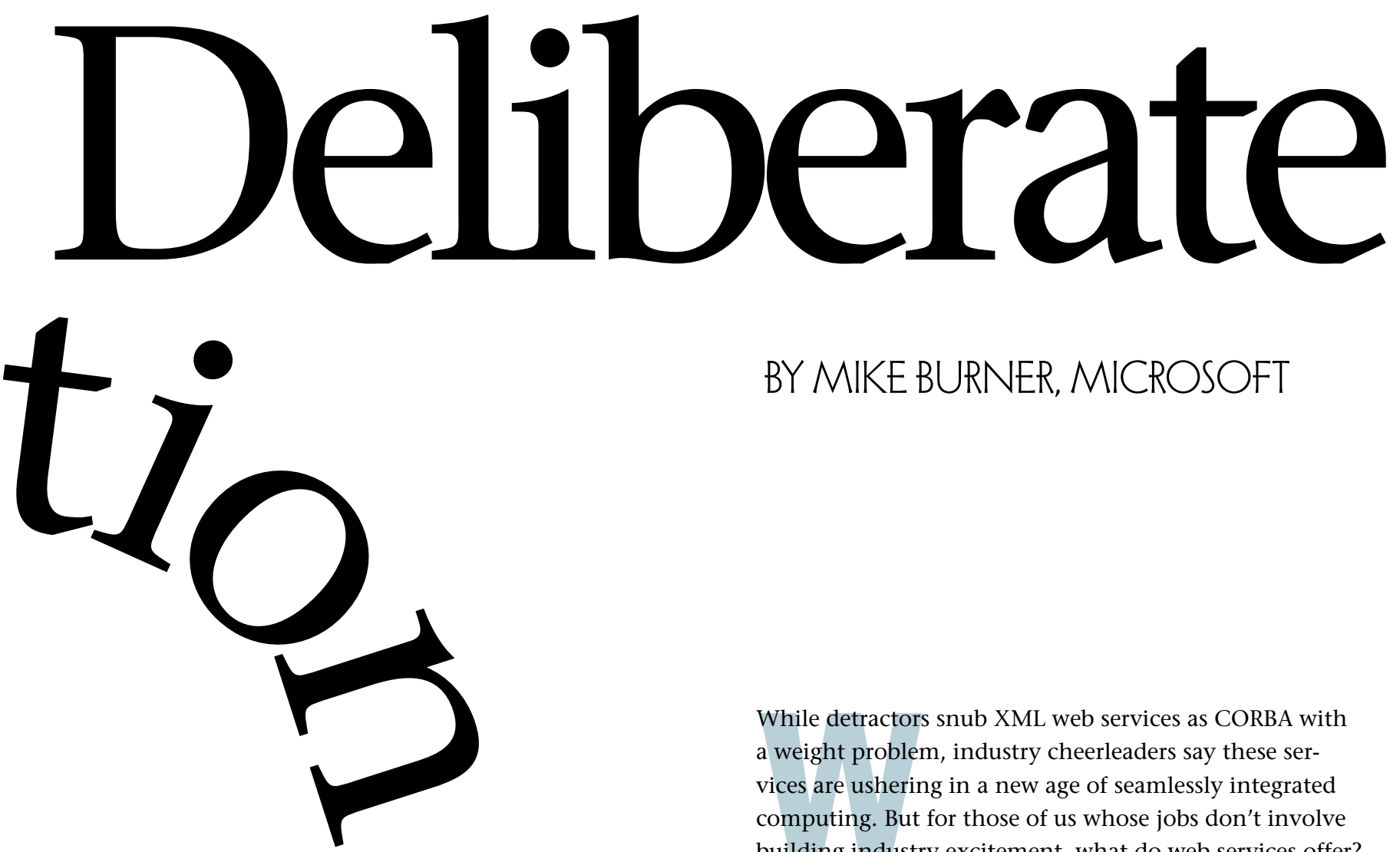

BY MIKE BURNER, MICROSOFT

While detractors snub XML web services as CORBA with a weight problem, industry cheerleaders say these services are ushering in a new age of seamlessly integrated computing. But for those of us whose jobs don't involve building industry excitement, what do web services offer?

The vast investment in Internet infrastructure and telecommunications over the past decade is making the previously-unthinkable eminently achievable. Organizations can now retrieve up-to-the-minute data at run-time, but must now agree on protocols for retrieving and updating data, and on means of demonstrating the privilege

Creating Connectedness with XML Web Services. to do so. Such necessities gave birth to XML web services, also known simply as web services, which attempt to bridge myriad Internet systems. These services have generated much excitement and vendors are scrambling to depict their platforms as the most compliant, mature, secure, or simply the most likely to crank out swell T-shirts. This article attempts to dive beneath the hype, examining how XML web services differ from existing architectures, and how they might help build customer solutions. Let's begin by describing features of XML web services. These services:

Expose Programmable Application Logic. Scenarios for calling a web service run from retrieving the phone number of a local restaurant to searching for extraterrestrial intelligence. Like a call to a class library or analytic engine, you use web services when you cannot, or would rather not, implement the logic yourself. 


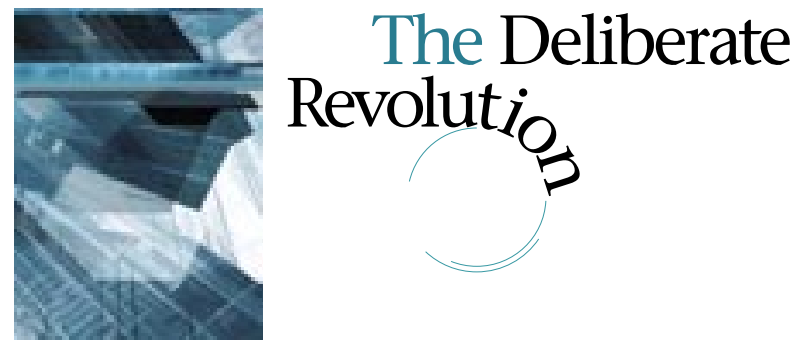

Are accessed using standard Internet protocols. At minimum, this means utilizing TCP/IP or UDP, but web services are usually exposed using HTTP operating on top of TCP.

Communicate by passing messages. A web service is defined by its messages, which must be self-sufficient, referencing information necessary to understand the message. In practice, part of this message state may be implied: when using HTTP, the service reply is interpreted as responsive to the request sent on the same connection.

Use XML to structure messages. XML (eXtensible Markup Language) is a mature technology for representing data as self-describing, platform-independent text. Self-describing data identifies itself using element and attribute names. Elements identify their type, such as "integer," using the XML Schema Definition Language (XSD). By using a common collection of data types, web services unambiguously translate service state between its wire representation and the platform-specific data types used for processing. This interoperability at the state level is the key to achieving integration using XML web services.

Package messages according the SOAP specification. "SOAP" is an acronym for "Simple Object Access Protocol," but that expansion implies a programming style inconsistent with the document-centric style growing dominant in the web services space.

SOAP is a simple protocol that defines a message structure to include an optional Header element and a mandatory Body element, wrapped by an Envelope element. While simple in itself, SOAP supports the creation of complex self-contained messages.

Describe themselves using WSDL. The Web Services Description Language (WSDL) allows a service to define messages it accepts and produces; collectively, these messages define the service contract. WSDL also permits the service to identify network endpoints that honor this contract.
Support their own discovery. If you can find a WSDL document, you can use the web service it describes. But how do you find the service on your local area network, rather than the one halfway around the world? UDDI (Universal Description, Discovery and Integration) supports both design-time and run-time discovery of web services. UDDI is implemented as a web service, and you run it within a trust domain. Several public implementations of UDDI provide an Internet-scale directory of businesses and services.

Are not remote procedure calls. Now to stir the pot a little: good web services are modeled as document retrievals and updates, not as remote procedure calls (RPCs). RPC is primarily designed to support method invocation between tightly bound but topologically distributed systems. The document-centric approach abstracts away the system architectures, creating loosely-coupled connectedness that withstands changes to the underlying implementations. You can still achieve interoperability while using "RPC/Encoded" SOAP messages, but the focus on objects and method invocation is contrary to the document-centric philosophy of web services. SOAP messages can be in two styles, "RPC" and "Document," and can use two serialization formats, "Encoded" and "Literal." In practice, SOAP-RPC uses "RPC/Encoded," while document-centric web services use "Document/ Literal."

Are not CORBA. Strong analogies exist between web services and CORBA, such as the respective roles of WSDL and IDL, but CORBA is fundamentally object-oriented. The document-style messages used in web services offer more flexibility. For example, an interception service might operate on a document header element, without understanding the rest of the document. As web service technologies evolve, standards bodies like W3C and IETF will help smooth the rough edges of specifications.

\section{TINY BUBBLES}

Over the next few years, we will experience a groundswell of innovation in two areas: The definition of XML 
<s:Envelope xmlns:s="http://schemas.xmlsoap.org/soap/envelope/">

$<$ s:Body $>$

$<$ thisApp:Order xmlns:thisApp="URI-1">

$<$ thisApp:Item>

$<$ partNumber $>1234567890</$ partNumber $>$

$<$ Quantity $>2</$ Quantity $>$

$</$ thisApp:Item>

<someStandard:deliveryAddress xmlns:someStandard="URI-2">

$<$ Name $>$ Jane Doe</Name>

$<$ Street1>1 Jane Doe Way</Street1>

$<$ City $>$ Nowhere $</$ City $>$

$<$ Province $>$ California $<$ /Province $>$

$<$ Country $>$ USA $</$ Country $>$

$<$ postalCode $>00000-0000</$ postalCode $>$

$</$ someStandard:deliveryAddress $>$

<anotherStandard:paymentInstrument xmlns:anotherStandard="URI-3">

$<$ instrumentType $>$ Visa $<$ /instrumentType $>$

$<$ instrumentID>0000000000000000</instrumentID>

<instrumentEvidence type="Owner" $>$ Jane Doe</instrumentEvidence $>$

$<$ instrumentEvidence type="expiryDate">01JAN2004</instrumentEvidence $>$

$</$ anotherStandard:paymentInstrument>

$</$ thisApp:Order $>$

$</ \mathrm{s}$ :Body $>$

$</ \mathrm{s}$ :Envelope $>$

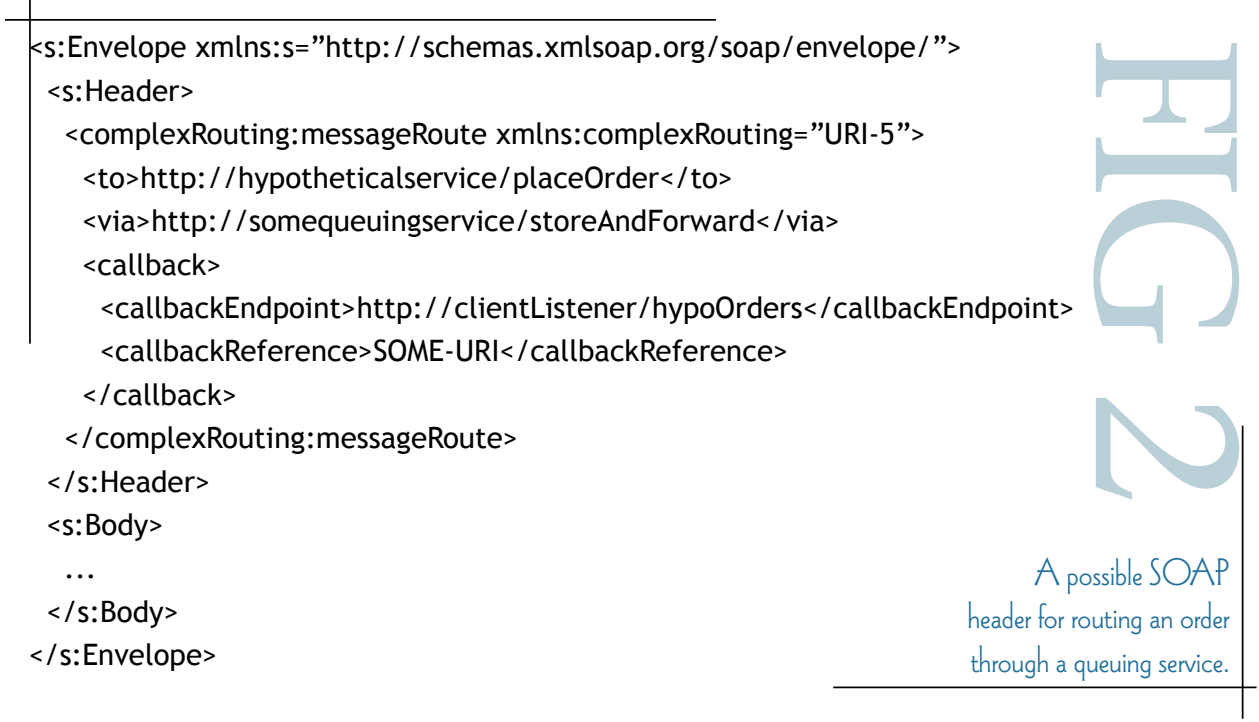

This hypothetical input message would satisfy basic requirements, without considering issues such as security and reliability. for a fictional purchasing service. This service must accept a document containing order, item, delivery address, and payment instrument elements; produce a SOAP Fault if input is incomplete or incorrect; and produce an order confirmation if the order is valid.

The input message depicted in Figure 1 does not need a Header element, since the functional requirements of the message can be represented in the message Body. But much of the effort in developing and deploying software solutions focuses on the operational requirements, such as security, reliability, and scalability. Let's look at how SOAP headers can be used to address these operational requirements.

A key operational requirement might be guaranteed delivery of messages. On the Internet, one cannot guarantee immediate (synchronous) delivery because of the inherent unreliability of networked systems. But a message queuing service can virtually guarantee asynchronous delivery (assuming the remote service comes back eventually). The client should push a message to the queuing service, receive acknowl-

edgement, and then disconnect. Once the process is complete, the order-processing service can route an order acknowledgement back to the client, using a callback endpoint provided by the client. This approach is called asynchronous messaging, and is an important service design pattern we will discuss later.

Figure 2 represents a possible SOAP header for routSOAP header by developing a hypothetical set of headers 


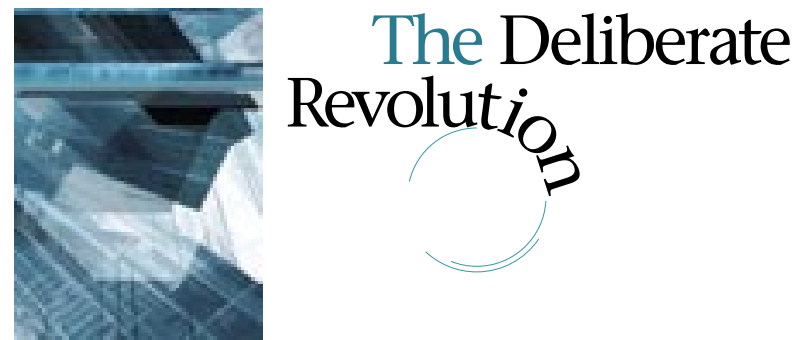

ing an order through a queuing service. The client will deliver the message to the queuing service and receive acknowledgement after the queuing service has persisted the message in its queue. The queuing service might insert another header element, instructing the order processing service to respond with a digitally signed acknowledgment. When the order processing is complete, the order-processing service will send a message-either an order acknowledgement or a SOAP fault-to the endpoint specified by the client; the protocol would no doubt require the callbackReference element to be returned, so the order originator could map the acknowledgement or exception to the correct order.

Reliable messaging solutions may need to retransmit unacknowledged messages until their receipt can be confirmed. This creates the possibility that the remote systems may receive the same message twice, but in the case of an order-processing service, this should not result in duplicate orders. When messages have an incremental effect on persistent state care must be taken to ensure idempotency: guaranteeing a single message has the same effect whether received once or multiple times. A SOAP header element can protect against incorrectly processing duplicate messages. A straightforward approach might include a client-generated globally unique identifier (GUID) in the request, as represented in Figure 3, which the server can store and check against.

Now imagine we want to make this transaction more

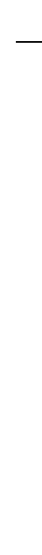

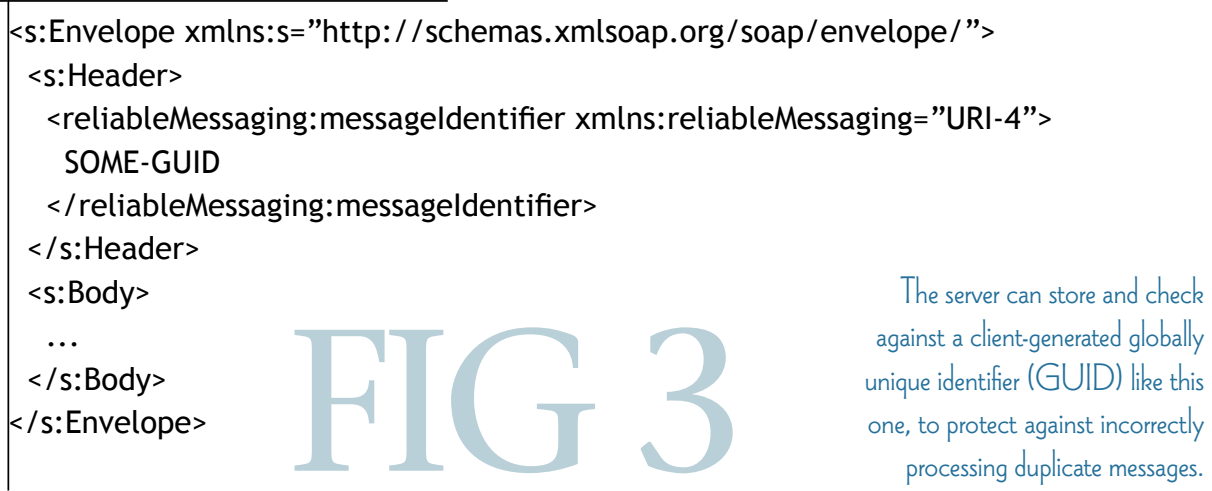

The server can store and check against a client-generated globally unique identifier (GUID) like this one, to protect against incorrectly processing duplicate messages.

secure. HTTPS might transmit the message, but every intermediary enroute would have to decrypt and encrypt the message again; this is computationally expensive and exposes confidential data to intermediaries. A solution is to encrypt the body so only the final destination can decrypt it, with the headers left unencrypted to facilitate processing, as illustrated in Figure 4.

Robust solutions to such challenges would be more complex in the real world as well as better factored; that is, the commonly usable header elements would be identified, defined separately, and embedded into other header elements. But these examples illustrate that SOAP Header extensions effectively attach metadata and operational instructions without disturbing the message body, and greatly simplify the development of an individual service, since many operational requirements can be satisfied by other services, or by a common message pipeline (explored later). Also, the self-contained nature of a SOAP message provides an opportunity to route it through one or more intermediaries, and to persist it for later processing, without losing context. The SOAP specification is likely to remain simple and flexible, with additions making it more extendable rather than more "complete."

\section{UNRESTFULNESS}

Since web service architecture is evolving so rapidly, is it ready for the real world? Do the XML/SOAP/WSDL standards contain fatal flaws? Is this author correct in asserting "document/literal" is the way to go, or is he saying so because he works for Microsoft? This section will explore what web services architecture potentially lacks, and conclusions will be left to the reader.

"Representational State Transfer" (REST) was coined by Roy Fielding to describe a reverseengineered view of web architecture. REST adher- 


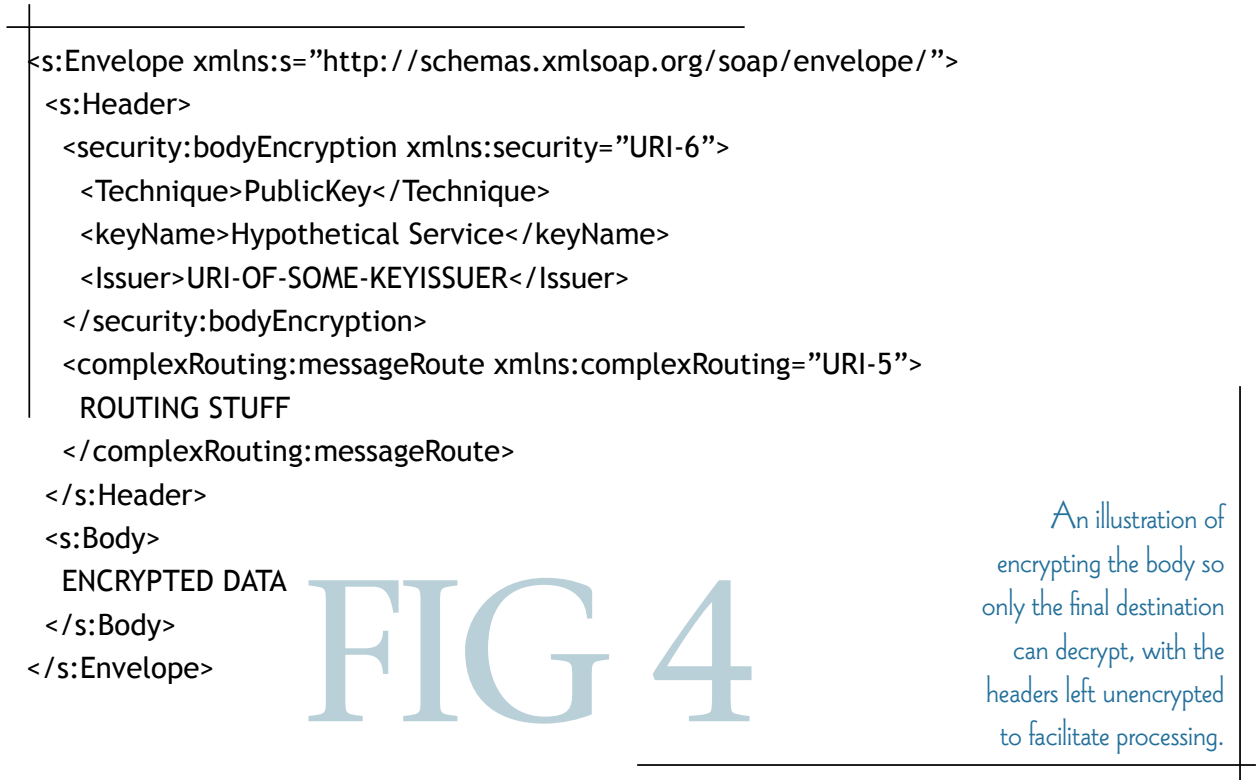

protocols: WSDL and XSD documents are typically referenced and retrieved using URLs and HTTP Get.

But why use SOAP when XML messages can be passed over HTTP, using mature Web protocols and infrastructure? What if the transport-agnostic format were just HTTP, wrapped by other transports as necessary? This would require the entire HTTP header be treated as part of the message and preserved accordingly. SOAP advocates would say the HTTP header

ents are sometimes misunderstood as antagonistic toward web services; on the contrary, they seek to leverage Web principles to make web services flexible enough to encompass a broad range of application requirements. The biggest concern in the REST camp is that the emergent web services architecture encourages the use of a single HTTP verb, Post, for all interactions. This subverts a strength of the Web architecture: that a proxy server can easily filter and route messages based on the four basic HTTP verbs, if they are used properly. Get should have no side effects to guarantee idempotency and promote caching. Post should be recognized as uncacheable and requiring special support to ensure idempotency. Put and Delete are absolutes and naturally idempotent, calling for responses not worth caching. (Note this view of idempotency ignores the effect on metadata, such as "time last modified.") Using Post to query web services also defeats URI addressability, which is commonly used for passing state from one step in a process chain to another.

The tenet that "Get should be used to query, Post to modify, and never the twain shall meet," has been violated since the Web's earliest days. Update requests are commonly coded into Get URLs for user experience purposes ("click here to confirm purchase"), and complex queries made using Post from Web forms. But past mistakes do not invalidate the principle.

The response of the SOAP camp is likely to be a set of header elements that clarify whether a message can be cached, resubmitted safely, and the like. But those header elements don't exist yet, and whether they will be supported by every network cache and proxy server is unknown. So, for now HTTP Get will not go away, which is also crucial to the functionality of the web services is clumsy and poorly designed for complex instructions. Modifying HTTP to support what SOAP does, the argument goes, is tantamount to recreating SOAP.

"Yawn," you say. "Of course, software architects are arguing with each other. It's their job. But do these things work?" Well, yes and no. Several limits of web service technologies exist, the greatest being the fragility of a solution dependent on Internet availability. No matter how carefully you design to avoid blocking on network requests, an application cannot progress if a critical service is unavailable. This issue needs to be addressed both organizationally and in service design.

Another issue is the uneven support for the document/literal style of messages in older SOAP toolkits. The first wave of SOAP usage focused on RPC scenarios. Several toolkits did a good job implementing RPC support, but a poor job supporting the document style. You should evaluate how your toolkit capabilities affect your ability to interoperate with partner organizations. Another problem is the platform immaturity. It makes no sense to solve problems like security, routing protocols, reliable messaging, and transactional support just to exchange invoices with suppliers.

Many are concerned the verbosity of XML, with all its namespace declarations and element names, will bog down connectivity. Fortunately, XML compresses well. Modern modems already compress text for transmission; expect to see similar techniques used on SOAP message bodies. A related concern is the performance implication of marshaling data as text (that is, converting binary data to text for transmission, then back into binary for processing); frankly, this is the price of interoperability.

Others are worried we will end up with a hodgepodge 


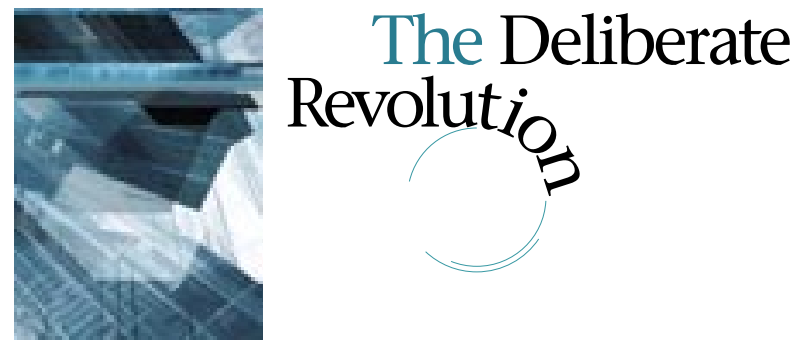

of standards and application servers that do not interoperate. As the old joke goes, the best thing about standards is there are so many to choose from. Technology vendors must make interoperability a top priority, and the marketplace and industry press must hold them to their promises. A promising development is the formation of the Web Services Interoperability Organization (WS-I), an industry consortium that defines protocol suite profiles against which web services technologies must demonstrate compliance.

Web services can certainly play an important role in your mission-critical systems, but for now they may need support from "non-native" infrastructure. You can get reliable messaging by routing through an MQSeries server; you can get secure communications with transport-level encryption; you can do authentication and authorization on existing systems. Over time, "native" solutions will take advantage of the graceful integration capabilities of web services architecture.

\section{SERVICE PATTERNS}

It's not perfect, but for many classes of problems the web services architecture is the most appropriate technology available. Web services are particularly useful for exposing state management services to a heterogeneous network of client components. Let's look at scenarios for deploying web services.

\section{1}

The Web Services Façade. Probably the most 1. common use of the façade pattern, which presents a "friendly" interface to "unfriendly" application logic, is to hide a call to an external resource that requires a different language or syntax than the rest of the application. The façade web service pattern is similar; it acts as an XML front-end to a service component that is not natively XML.

Think carefully about your implementation of authentication, authorization, and user impersonation when implementing a façade. If calls back to the original service use a privileged account (such as the Unix super user), it will defeat any authorization checks the service would normally provide, so the façade must reimplement those checks and be tested carefully. Façades such as XML front-ends to database services and business applications will abound while the industry transitions towards native XML interfaces.

\section{Exposing data behind the Web. One prom- • ise of web services is to create a "Programmable} Web." It follows, then, that web services will expose data and functionality currently supported via web pages. The essential difference is that data will be leveraged to go beyond information "pictures" available through a browser. Exposing web data allows partners and customers to embed service functionality into interfaces. Rather than forcing partners to "screen scrape" HTML to find the relevant data, XML gives them a stable, self-describing set of interfaces.

2 Interception. The interception pattern mediates 3 . between the client and the ultimate web service provider through routing, redirection, or retransmission. With routing, the client sends the message to the interception service based on prior instruction via local configuration or by run-time referral. Redirection would be a feature of a transport protocol such as HTTP. Retransmission would be performed by the receiving service, with the message sent to one or more other services before final processing.

The interception service we see on today's Web is the proxy server, which "intercepts" web traffic to provide security and caching services. With web services, we can expect to see services that push programmable functionality out to "virtual server farms" that embody the concept of "computing on tap." The interception pattern might also be used by an authentication service. Versioning web services might include dynamically routing old-format requests through a network endpoint capable of translating requests into new formats.

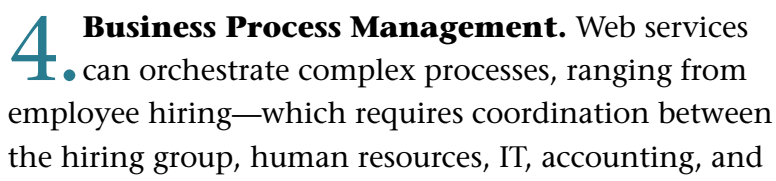


payroll—to auto insurance claims processing — which crosses the organizational trust boundaries of the insurance company, claims adjusters, repair shops, and often medical service providers and the police.

One challenge in process management involves handling updates to service state. The traditional art in transaction management focuses on locking the affected rows prior to an update, but this approach does not work in an environment of loose trust, high-latency connections, and unreliable transmissions. Interesting work is being done in the area of long-running transactions (also known as "sagas"), involving provisional commitment and compensatory actions that are invoked if the transaction fails or is canceled.

Catalog Publication. A product and service cata• $\log$ is probably the most flexible data feed a business can offer customers, partners, and sales channels. Sales team members can have it at their fingertips via laptops during customer visits. Support staff can update it as a common reference with customers. The organizational website can render XML into HTML for current and prospective customers.

For several reasons, catalogs can benefit from service agents: client code that facilitates access to your web services. First, the data tends to be relatively stable, so intelligent caching benefits both service scalability and user experience interactivity. Second, the data is often complex, so helping clients with common manipulation scenarios will increase service exploitation. The biggest challenge in publishing a catalog as a web service is in defining schema. The best strategy is to leverage what already exists in the vertical market segment and extend it as compatibly as possible.

Information Portals. Since the Web's beginnings, "portals" and "dashboards" have delivered valuable services ranging from consumer news sites, such as My Yahoo! and The Motley Fool, to network management sites developed by IT departments. Web services offer a new approach to these portals by defining a common schema for exchanging data. By exposing data through self-describing web services, the data publisher lets clients consume data without incurring the support costs of transmitting schema and interface instructions out-of-band to the client solution developer. This scenario relates to the earlier section on exposing web data. Intranet and extranet portals don't suffer from the business-model issues described in that section, but they do introduce more complex concerns around authorizing access to compartmentalized organizational information.
7 EAI Hubs. Enterprise Application Integration (EAI) - is the traditionally expensive process of constructing data interchange systems between business applications. EAI solutions must understand the protocols and service interfaces of the applications they manage, extract data from different applications, and translate data into schemas and types understood by the other applications. An effective model for EAI solutions is the hub. The hub typically translates extracted data into an internal representation, from which it can produce representations understood by all applications it services. A hub may use a connector approach to modularly add supported applications to an installation.

In the web services world, internal representation of data is a set of XML documents, and the connectors are web service façades that produce those documents. It would be overly-optimistic, however, to believe the problem ends there. Until application vendors commit to common schemas for business data, and build XML interfaces capable of supporting those schemas, business data translation will remain error-prone.

\section{WEB SERVICE DESIGN}

It's a good idea to start small, taking a "crawl, walk, and run" approach to building organizational credibility for web service architecture. Crawling might consist of building a web service within your organization. Pick a project with an awkward solution-perhaps an information service requiring users to run a terminal emulator and use arcane commands. Build a web services façade in front of the application and expose the functionality through a web page or a smart client application. Walking might consist of exposing read-only data of an order-status service using web services. You may not "run" for some time yet, depending on how quickly key infrastructure services, such as inter-domain authentication mechanisms, are standardized.

Web services will make XML schemas-now the obscure domain of your database architect-central to how organizations communicate. Web services work best when they support your internal processes and your external data view. A consistent view of the information driving your business guarantees you and your customers will stay in sync. Start by researching what schema work is ongoing in your industry. Get involved in the process to make sure your organization's interests are represented, and good design principles are followed. Good XML schemas are:

- General purpose. Create generally useful schemas and use derived schemas to add constraints where needed. 


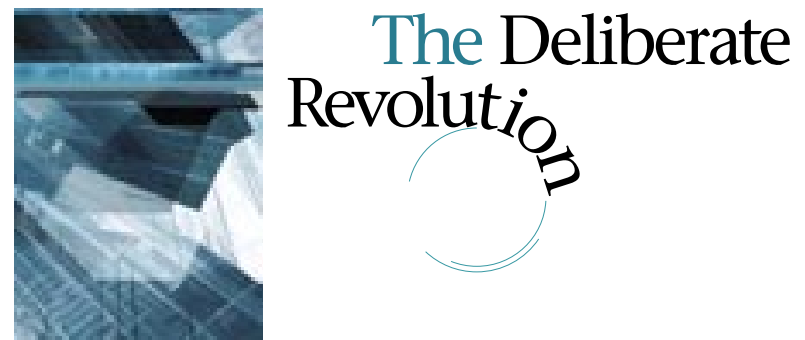

When modeling interfaces to your data elements, treat them like the document fragments they are. Start with the basic interfaces of Query, Update, Add, and Delete, and use element-specific methods only as necessary.

- Platform agnostic. Use the XSD data types and complex types built from the XSD types.

- Standard observant. If a generally accepted schema for a telephone number exists, use it. Do not invent a new one; do not treat telephone numbers as strings. Design for interoperability.

- Extendible. Anticipate the need for applications to "decorate" data with custom properties, and design elements that allow it. One approach might be an "<extension>" element with an XSD "any" type to permit applications to embed XML snippets into your data elements.

- Usable. Think hard about error cases, and define your SOAP faults to be meaningful to the client. Log all fault responses and analyze the logs for failure patterns.

Designing for Security and Reliability. As you work up to a run with web services, you will need to develop the sophistication of your authentication system. Your early internal services may simply use your existing domain access systems, such as NIS or Active Directory. But when developing services that authenticate external users, avoid creating and managing identities for them. Authenticate users from partner organizations using the credentials managed by the partner. Look at the WS-Security specification and related activities to understand how those credentials can be attached to SOAP messages and verified. Consumer services should leverage identities provided by the user's ISP, or by Internet-scale authentication services. There is considerable activity in the latter space, including the Liberty Alliance and Microsoft's Passport, but much work is needed to make "universal single sign-on" a reality.

Your web services need to support several types of access. The best way to implement granular authorization is through role-based security. For each web service, determine how data will be accessed and modified, and to what roles these activities map. For example, a web service that manages a calendar might give full-access to the calendar owner; allow designated individuals to add and delete work-related commitments; allow co-workers to see but not modify, work-related activities; and allow authenticated external partners to see when users are available for meetings.

Well-run web services within your organizational network should present few reliability concerns, but services accessed over the Internet offer greater challenges. A good policy is not to block on service access. Make sure you have a control thread to clean up unresponsive requests and take compensatory action. The "thread pool" - in which several worker threads are kept available to "wake up" and manage high-latency work items-is a good design pattern for accessing web services.

Expect access failure. Intelligently manage retries of failed requests, being careful to consider the implications of repeating requests that incrementally update service state. Design processes to fail gracefully while notifying the appropriate people who should investigate the failure. Provide explicit caching instructions on the documents your service produces so client components can reduce their service requests. Finally, be reasonable in how you use web services. The value of the service must justify the latency hit of accessing it.

Versioning web services. As with any component-programming model, client applications will bind to specific versions of web services. Microsoft Windows ${ }^{\mathrm{TM}}$ famously suffers from "DLL hell," where updates to shared libraries via newly installed applications break older applications bound to earlier library versions. Take care not to create "XML hell." XML schemas and WSDL documents must be updated using a versioning scheme that avoids breaking existing clients. The best way to do this is by creating the new schemas and contracts in unique namespaces.

In an XML schema, the namespace is specified using the "targetNamespace" attribute of the "schema" element. In a WSDL document, use the "targetNamespace" attribute of the "definitions" element. One approach might be to encode traditional version numbers into a URI: targetNamespace="http://orgname/serviceName/ 
versionNumber." When you publish a new version of the service, do so in a new namespace created by incrementing the version number. To avoid having to maintain multiple implementations of your service logic, consider using the "web service façade" pattern described earlier to translate old-format requests and responses.

Design Guidelines. The unique strengths and limitations of web services suggest unique design patterns, including:

- Large granularity messages. Because of their high latency, web services should not be designed with "chatty" protocols. Do not design services to expose interfaces making small updates to data elements; rather, use generic "update" methods that can accept a transformed element in a single service call.

- Asynchronous messaging. For services with indeterminate latency, it is good practice to post the request, use the response as simple acknowledgement of receipt, disconnect, and the client can poll the server for completion. The next bullet describes a better design for complex processes.

- Bi-directionality of services. It is often useful to implement web service pairs to manage the request-response loop. A library consortium might provide a service called PostBookRequest, which can be called by a member library to request a book. The member library might host a service called PostBookRequestResponse, where the consortium system sends updates on the acquisition progress.

- Endpoint discovery. Sometimes it's desirable for the client to select the best endpoint at run-time rather than hard-coding endpoints. Examples include dynamic load balancing, fail-over when primary systems go down, and achieving topological or geographic affinity between the client and the service instance. A long-running client might find a service instance every time it starts up; another client might cache service-binding information.

- Idempotency. Protect against the mishandling of messages received more than once because of network problems. As discussed earlier, the main danger is messages incrementally affecting service state, such as requests to purchase. When working with such messages, design in unique identifiers that can identify duplicate messages.

- Service agents. You may wish to give client component developers code that optimizes access to your web service. Scenarios for using service agents include the performance of complex integrity checking so poorly formed requests can be repaired without a roundtrip to the server, and support for intelligent caching of service data to reduce roundtrips to the service.

- Request pipeline. As your organization broadens its use of web services, you will find yourself running requests through a common set of processes to "unwind" the SOAP header, validate authentication credentials, check authorization to access the interface, and log activity. Pipeline code can be deployed as shared libraries called by the service implementation, or as an interception service that passes the validated and transformed request across a trusted interface to the service-specific code.

- Context and content-based routing. Web service implementations may be distributed across many physical devices hosted in multiple data centers. Service logic can route requests to specific service instances, based on any message element or attribute.

\section{READY OR NOT, HERE THEY COME}

Common schema for describing interactions will unblock information flow between organizations, and allow us to communicate with unprecedented precision. But getting there requires fresh thinking on data and process management. Web services, which promise to deliver to intercomponent communications the open connectivity the Web has enabled for person-to-person communications, will be central to every facet of the transformation. $Q$

MIKE BURNER (mburner@microsoft.com) works on web services as software architect at Microsoft. Mike's recent work includes collaboration technologies, web-based storage services, and participation in the specification of .NET My Services. Prior to Microsoft, Mike worked at Alexa Internet developing web-profiling technologies, web service-based syndication technologies, and the Internet Archive. Mike's fascination with integration and interoperability comes from his early career as a Unix systems programmer and system manager at Harvard University and Xerox, where his days were spent getting Unix flavors, VMS, CMS, Macs, PCs, and Xerox Network Services to play nicely with each other. 